\title{
Checklist de ostrácodes (Crustacea, Ostracoda) dulcícolas do Pantanal Sul Mato-grossense, Brasil
}

\author{
Janet Higuti', Kennedy Francis Roche ${ }^{2} \&$ Koen Martens ${ }^{3,4}$
}

\begin{abstract}
1. Universidade Estadual de Maringá, Nupelia, PEA, Av. Colombo, 5790, 87020-900 Maringá, PR, Brasil. (janethiguti@gmail.com) 2. Universidade Federal do Mato Grosso do Sul, Cidade Universitária, Caixa Postal 549, 79070-900 Campo Grande, MS, Brasil.

3. Royal Belgian Institute of Natural Sciences, Freshwater Biology, Vautierstraat 29, B-1000 Brussels, Belgium.

4. University of Ghent, Biology, K. L., Ledeganckstraat 35, B-9000 Ghent, Belgium.
\end{abstract}

Recebido 5 dezembro 2016

Aceito 6 fevereiro 2017

DOI: 10.1590/1678-4766e2017114

\begin{abstract}
Checklist of freshwater ostracods (Crustacea, Ostracoda) of the Pantanal of Mato Grosso do Sul, Brazil. Nowadays there are about 2,000 species of non-marine Ostracoda described in diverse aquatic environments in the world, of which 117 species from 37 genera are known in Brazil. The present survey of ostracods of the Pantanal of the state of Mato Grosso do Sul, recorded 35 species belonging to the families Cyprididae, Candonidae, Limnocytheridae and Darwinulidae. The ostracod fauna of Brazil is still poorly known and the ostracod biodiversity from several habitats (e.g. interstitial) remains unknown. In this sense, there is a great probability to find new records and new taxa of ostracods in the Pantanal, as well as throughout Brazil.
\end{abstract}

KEYWORDS. Crustaceans, floodplain, taxonomy, Biota-MS Program.

RESUMO. Atualmente existem cerca de 2.000 espécies de Ostracoda dulcícolas descritas em diversos ambientes aquáticos do mundo, das quais cerca de 117 espécies em aproximadamente 37 gêneros são conhecidas no Brasil. O presente levantamento de ostrácodes do Pantanal Sul Mato-grossense, registrou 35 espécies pertencentes às famílias Cyprididae, Candonidae, Limnocytheridae e Darwinulidae. A fauna de ostrácodes do Brasil ainda é pouco conhecida e a biodiversidade de ostrácodes de vários habitats (por exemplo, instersticial) ainda permanecem desconhecidos. Neste sentido, existe grande probabilidade de se encontrar novos registros e novos táxons de ostrácodes no Pantanal, assim como em todo território brasileiro.

PALAVRAS-CHAVE. Crustáceos, planície de inundação, taxonomia, Programa Biota-MS.

O Pantanal mato-grossense é uma das maiores áreas úmidas contínuas do planeta, um bioma único qualificado pela alta produtividade biológica que se reflete na elevada abundância da vida selvagem. A sua importância para a conservação da biodiversidade aquática e para a manutenção das funções do ecossistema aquático é amplamente reconhecida, em virtude da criação da Reserva da Biosfera e Patrimônio da Humanidade. Apesar deste grande valor sócio-econômico e científico, bem como as evidências de impactos antropogênicos crescentes (FERREIRA et al., 1994; POR, 2000; SwARTS, 2000), ainda existem várias lacunas sobre o conhecimento da biodiversidade de diversas comunidades biológicas, entre as comunidades aquáticas destacam-se os ostrácodes.

Os ostrácodes são pequenos crustáceos, com comprimento variando de 0,3 a $3 \mathrm{~mm}$, caracterizados por apresentar uma carapaça bivalvada que engloba totalmente o corpo e os apêndices (HoRne et al., 2002). São muito comuns nos corpos de água interiores, e abundantes nos bentos e associados à vegetação emersas e submersas, mas também ocorrem em ambientes marinhos, intersticiais e ainda em ambientes terrestres e semiterrestres. Os ostrácodes são interessantes objetos de estudo da mudança da biodiversidade, pois suas valvas calcificadas podem formar microfósseis abundantes em sedimentos de lagoas. O estrato de sedimento pode ser analisado e alterações em densidades e vários outros aspectos da diversidade podem ser analisados sobre molduras de tempo real.

Ostracoda são divididos em duas subclasses, Myodocopa e Podocopa (Horne et al., 2002). Podocopa, por sua vez, está dividida em três ordens, seis subordens e oito superfamílias. Apenas três dessas superfamílias, Cypridoidea, Cytheroidea e Darwinuloidea, possuem representantes em ambientes dulcícolas.

Atualmente existem cerca de 2.000 espécies de Ostracoda descritas em aproximadamente 200 gêneros em diversos ambientes não marinhos no mundo, das quais cerca de 275 espécies são conhecidas em 55 gêneros na América do Sul e Central (Martens \& Behen, 1994; Martens et al., 2008). Considerando que esta é uma subestimação na escala global, a América do Sul seria um exemplo, e mais ainda o Brasil. MARTENs et al. (1998) relataram apenas 96 espécies descritas em 32 gêneros em nosso país. As recentes adições à fauna brasileira de ostrácodes realizadas nos estados do Rio Grande do Sul (Würdig \& PINTO, 2001); São Paulo (PINTO et al., 2003, 2004, 2005a,b, 2008) e Paraná/Mato Grosso do 
Sul (Higuti et al., 2009a; Higuti \& MARTens, 2012a, b, 2014, Higuti et al., 2013) elevaram esse número para 117 espécies em 37 gêneros. No entanto, novos gêneros e novas espécies ainda aguardam descrições nos diferentes ambientes (Higuti, 2006; Pinto, 2007; Higuti et al., 2009b, 2010).

\section{MATERIAL E MÉTODOS}

A lista de espécies foi baseada no material amostrado na estação seca (junho de 2003), durante o projeto "Biodiversidade de microbiota de ecossistemas aquáticos do Pantanal de Nhecolândia 2003-2004", além da consulta do artigo "Preliminary comparative data on the fauna of the pleuston in the southern Pantanal, Brazil, with emphasis on the microcrustaceans", realizado por RocHA \& POR (1998) em ambas as estações, chuva (fevereiro/março de 1994) e seca (julho de 1994).

\section{RESULTADOS E DISCUSSÃO}

Lista das espécies do Pantanal Sul Mato-grossense. A Figura 1 e a Tabela I mostram a localização e as respectivas coordenadas dos ambientes amostrados em junho de 2003. O estudo realizado por RocHA \& POR (1998) foi conduzido no Pantanal Sul, em uma área drenada pelos rios Miranda, Abrobal, Negro e Taquari, próxima às confluências com o rio Paraguai.

No Apêndice I consta a lista de espécies de ostrácodes de água doce registradas no Pantanal Sul Mato-grossense.

Comentários sobre a lista, riqueza do estado comparado com outras regiões. Entre os crustáceos de água doce, cladóceros e copépodos têm sido relativamente bem explorados em nosso país, tanto em termos ecológicos como em estudos sobre taxonomia. Por outro lado, a despeito de os ostrácodes serem abundantes na maioria dos corpos de água, ainda existem poucos estudos em ambientes aquáticos continentais brasileiros (WÜRDIG \& FREITAS, 1988; RoCHA \& Por, 1998; Higuti, 2006; Higuti et al., 2007, 2009b, 2010; Miranda, 2008; Mormul et al., 2010; Pereira, 2010; Matsuda, 2011; Chaves 2012; Matsuda et al. 2015a,b; Higuti \& Martens, 2016).

Foram registradas 35 espécies pertencentes a Cyprididae, Candonidae, Limnocytheridae e Darwinulidae (Apêndice I). Cyprididae foi a mais especiosa (26 espécies); esta família pode compreender acima de $80 \%$ de todas as espécies de água doce, na maioria das regiões tropicais (MARTEns, 1998). Em uma escala global, Cyprididae abrange cerca de 1.000 espécies, mais de metade de todas as espécies dulcícolas descritas de ostrácodes (MARTENs et al., 2008).

A riqueza de espécies de ostrácodes nos 28 ambientes do Pantanal Sul Mato-grossense (35 espécies) foi inferior ao registrado nos 48 ambientes do vale aluvial do alto rio Paraná (54 espécies) (Higuti et al., 2010), no entanto, o valor de riqueza no Pantanal foi superior ao observado na lagoa Emboada no Rio Grande do Sul (15 espécies) (WüRDIG \& FREITAS, 1988) e lagoas Jacaré e Águas Claras na bacia do Rio Doce do Estado de Minas Gerais (11 espécies) (MirandA,

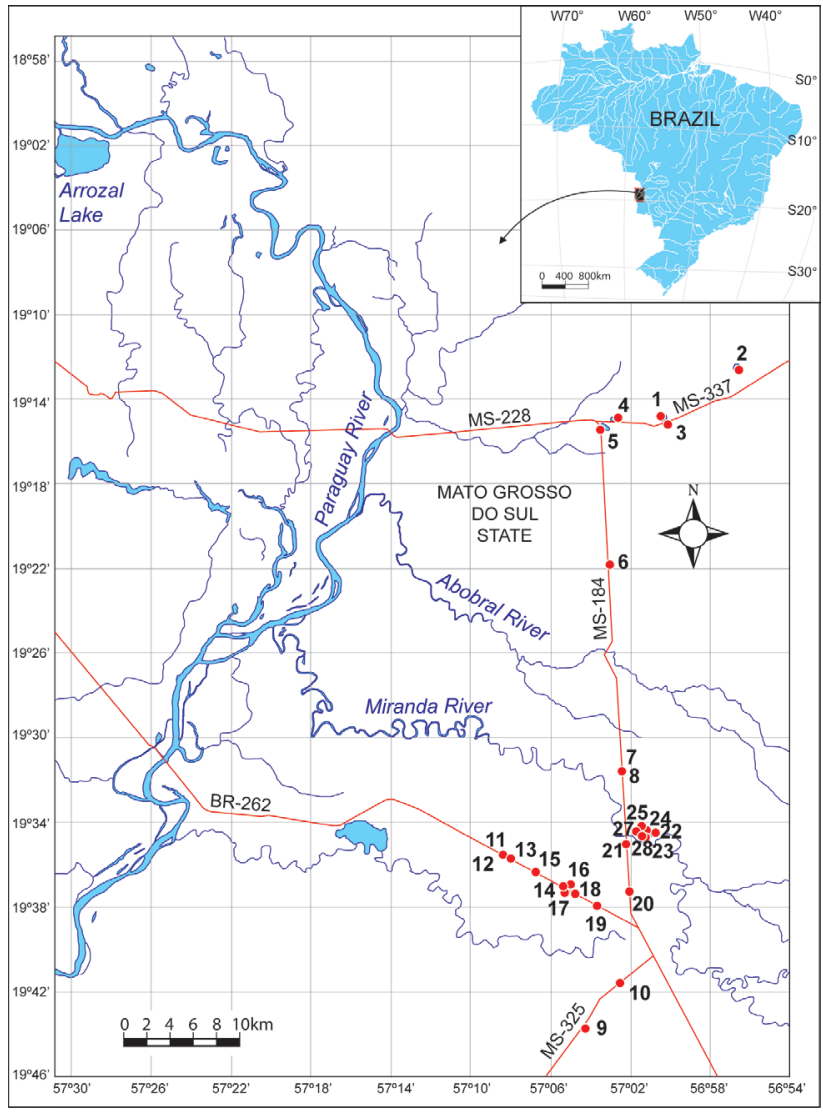

Fig. 1. Localização dos ambientes amostrados em junho de 2003 no Pantanal Sul Mato-grossense, Brasil.

Tab. I. Localidade e coordenadas dos ambientes amostrados em junho de 2003 no Pantanal Sul Mato-grossense, Brasil.

\begin{tabular}{|c|c|c|}
\hline Localidade & \multicolumn{2}{|c|}{ Coordenadas } \\
\hline 1. Baía São Miguel & $19^{\circ} 14^{\prime} 57^{\prime \prime} \mathrm{S}$ & $57^{\circ} 00^{\prime} 21^{\prime \prime} \mathrm{W}$ \\
\hline 2. Baía da Reserva & $19^{\circ} 12^{\prime} 32^{\prime \prime S}$ & $56^{\circ} 56^{\prime} 34^{\prime \prime} \mathrm{W}$ \\
\hline 3. Lecque Road pool 1 & $19^{\circ} 15^{\prime} 11^{\prime \prime S}$ & $57^{\circ} 00^{\prime} 13^{\prime \prime} \mathrm{W}$ \\
\hline 4. Lecque Road lake & $19^{\circ} 15^{\prime} 06^{\prime \prime} \mathrm{S}$ & $57^{\circ} 02^{\prime} 49^{\prime \prime} \mathrm{W}$ \\
\hline 5. CD Road lake 1 & $19^{\circ} 15^{\prime} 29^{\prime \prime} \mathrm{S}$ & $57^{\circ} 03^{\prime} 28^{\prime \prime} \mathrm{W}$ \\
\hline 6. CD Road lake 2 & $19^{\circ} 21^{\prime} 51^{\prime \prime S}$ & $57^{\circ} 03^{\prime} 04^{\prime \prime} \mathrm{W}$ \\
\hline 7. CD Road lake 3 & $19^{\circ} 31^{\prime} 34^{\prime \prime} \mathrm{S}$ & $57^{\circ} 02^{\prime} 27^{\prime \prime} \mathrm{W}$ \\
\hline 8. CD Road lake 4 & $19^{\circ} 31^{\prime} 34^{\prime \prime} \mathrm{S}$ & $57^{\circ} 02^{\prime} 27^{\prime \prime} \mathrm{W}$ \\
\hline 9. CF Road Lake 1 & $19^{\circ} 43^{\prime} 39^{\prime \prime S}$ & $57^{\circ} 04^{\prime} 19^{\prime \prime} \mathrm{W}$ \\
\hline 10. CF Road lake 2 & $19^{\circ} 41^{\prime} 33^{\prime \prime S}$ & $57^{\circ} 02 ’ 34^{\prime \prime} \mathrm{W}$ \\
\hline 11. CR lake 1 & $19^{\circ} 35^{\prime} 33^{\prime \prime S}$ & $57^{\circ} 08^{\prime} 19^{\prime \prime} \mathrm{W}$ \\
\hline 12. CR pool 1 & $19^{\circ} 35^{\prime} 33^{\prime \prime} \mathrm{S}$ & $57^{\circ} 08^{\prime} 19^{\prime \prime} \mathrm{W}$ \\
\hline 13. CR pool 2 & $19^{\circ} 35^{\prime} 38^{\prime \prime} \mathrm{S}$ & $57^{\circ} 08^{\prime} 03^{\prime \prime} \mathrm{W}$ \\
\hline 14. CR lake 2 & $19^{\circ} 37^{\prime} 09^{\prime \prime} \mathrm{S}$ & $57^{\circ} 05^{\prime} 11^{\prime \prime} \mathrm{W}$ \\
\hline 15. CR pool 3 & $19^{\circ} 36^{\prime} 17^{\prime \prime} \mathrm{S}$ & $57^{\circ} 06^{\prime} 46^{\prime \prime} \mathrm{W}$ \\
\hline 16. CR lake 3 & $19^{\circ} 37^{\prime} 11^{\prime \prime S}$ & $57^{\circ} 05^{\prime} 01^{\prime \prime} \mathrm{W}$ \\
\hline 17. CR lake 4 & $19^{\circ} 37^{\prime} 13^{\prime \prime S}$ & $57^{\circ} 05^{\prime} 03^{\prime \prime} \mathrm{W}$ \\
\hline 18. CR pool 4 & $19^{\circ} 37^{\prime} 14^{\prime \prime} \mathrm{S}$ & $57^{\circ} 04^{\prime} 56^{\prime \prime} \mathrm{W}$ \\
\hline 19. CR lake 5 & $19^{\circ} 37^{\prime} 53^{\prime \prime S}$ & $57^{\circ} 03^{\prime} 43^{\prime \prime} \mathrm{W}$ \\
\hline 20. CD Rd lake 5 & $19^{\circ} 37^{\prime} 15^{\prime \prime} \mathrm{S}$ & $57^{\circ} 02^{\prime} 04^{\prime \prime} \mathrm{W}$ \\
\hline 21. CD Rd, cattle pond & $19^{\circ} 34^{\prime} 55^{\prime \prime S}$ & $57^{\circ} 02^{\prime} 14^{\prime \prime} \mathrm{W}$ \\
\hline 22. Medalha lake & $19^{\circ} 34^{\prime} 32^{\prime \prime S}$ & $57^{\circ} 00^{\prime} 51^{\prime \prime} \mathrm{W}$ \\
\hline 23. BEP wetland 1 & $19^{\circ} 34^{\prime} 38^{\prime \prime} \mathrm{S}$ & $57^{\circ} 01^{\prime} 11^{\prime \prime} \mathrm{W}$ \\
\hline 24. BEP wetland 2 & $19^{\circ} 34^{\prime} 38^{\prime \prime} \mathrm{S}$ & $57^{\circ} 01 ' 11^{\prime \prime} \mathrm{W}$ \\
\hline 25. Camp Rd pool 1 & $19^{\circ} 34^{\prime} 25^{\prime \prime} \mathrm{S}$ & $57^{\circ} 01^{\prime} 299^{\prime} \mathrm{W}$ \\
\hline 26. Camp Rd pool 2 & $19^{\circ} 34^{\prime} 30^{\prime} \mathrm{S}$ & $57^{\circ} 01^{\prime} 23^{\prime \prime} \mathrm{W}$ \\
\hline 27. Camp Rd pool 3 & $19^{\circ} 34^{\prime} 28^{\prime \prime} \mathrm{S}$ & $57^{\circ} 01^{\prime} 16^{\prime \prime} \mathrm{W}$ \\
\hline 28. Camp Rd pool 4 & $19^{\circ} 34^{\prime} 34^{\prime \prime} \mathrm{S}$ & $57^{\circ} 01^{\prime} 07^{\prime \prime} \mathrm{W}$ \\
\hline
\end{tabular}


2008). Os primeiros inventários da fauna de ostrácodes dos hábitats aquáticos do Pantanal registraram uma elevada riqueza de ostrácodes, e pelo menos um gênero e quatro espécies são novos para a ciência. Este é um exemplo do pouco conhecimento sobre a biodiversidade desse grupo, tendo em vista as extensas áreas brasileiras, com uma rica fauna aquática que permanece inexplorada ou pobremente investigada em relação à fauna de ostrácodes de água doce.

Neste sentido, o projeto em andamento SISBIOTA/ CNPq/Fundação Araucária desenvolvido no NUPELIA/PEA/ UEM por vários pesquisadores brasileiros e estrangeiros, teve uma iniciativa única abordando a biodiversidade aquática em grande nível regional, comparando a biodiversidade de diversos grupos de organismos (incluindo Ostracoda) entre quatro grandes várzeas brasileiras, ou seja, Paraná, Pantanal, Araguaia e Amazônia.

Principais grupos de pesquisa. Dra. Janet Higuti (Núcleo de Pesquisas em Limnologia, Ictiologia e Aquicultura - NUPELIA, Universidade Estadual de Maringá, Maringá, PR); Dr. Koen Martens (Royal Belgian Institute of Natural Sciences, Bruxelas, Bélgica); Dra. Norma Luiza Würdig (Departamento de Zoologia, Universidade Federal do Rio Grande do Sul - UFRGS, Porto Alegre, RS); Dr. Ricardo Lourenço Pinto (Instituto de Geociências, Universidade de Brasília - UnB, Brasília, DF).

Principais acervos do Pantanal. Museu de Zoologia da Universidade de São Paulo (MZUSP), São Paulo, SP; Coleção de Ostracoda da Royal Belgian Institute of Natural Sciences (OC), Bruxelas, Bélgica e Laboratório de Ecologia de Macroinvertebrados do Núcleo de Pesquisas em Limnologia, Ictiologia e Aqüicultura (NUPELIA) da Universidade Estadual de Maringá (UEM), Maringá, PR.

Principais lacunas de conhecimento. A fauna de ostrácodes do Brasil ainda é pouco conhecida (MARTENS \& BEHEN, 1994) e vários hábitats, como intersticial, lagoas temporárias, riachos, reservatórios, ambientes terrestres, entre outros, permanecem desconhecidos. Apesar do grande esforço para conhecer a biodiversidade de ecossistemas aquáticos, torna-se necessário maior investigação no que se referem aos ostrácodes nestes diferentes habitats. Além disso, deve-se considerar a importância do monitoramento anual. Higuti et al. (2013) utilizaram pela primeira vez análise molecular para resolver problemas taxonômicos sobre os ostrácodes brasileiros. Recomenda-se fortemente que tais métodos sejam mais utilizados, como estes podem confirmar espécies morfológicas e revelar o potencial da diversidade críptica (BoDE et al., 2010).

Uma outra lacuna está relacionada ao pouco conhecimento sobre a ecologia (ocorrência, distribuição, etc.) e biologia (ciclo de vida, alimentação, comportamento, etc) de grande parte das espécies. Além disso, a ausência de chaves de identificação de ostrácodes, aliada a poucos especialistas no Brasil, dificultam a formação de novos profissionais que se dediquem à taxonomia e ecologia destes crustáceos.

Perspectivas de pesquisa em Ostracoda para os próximos 10 anos. Os resultados do projeto SISBIOTA/ $\mathrm{CNPq} /$ Fundação Araucária, o qual abrange quatro planícies de inundação brasileira, irão contribuir para o melhor conhecimento e incremento da biodiversidade de Ostracoda em nosso país. Além disso, um checklist de ostrácodes do Brasil, além de um atlas de identificação está sendo elaborado, com informações sobre distribuição e alguns aspectos ecológicos das diferentes espécies.

Trabalhos futuros sobre o conhecimento taxonômico de Cypridopsinae e Candonidae, além de estudos direcionados as análises moleculares para compreender as espécies complexas, e incremento de pesquisas com ostrácodes terrestres.

Agradecimentos. À Fundação de Apoio ao Desenvolvimento do Ensino, Ciências e Tecnologia do Estado de Mato Grosso do Sul (Fundect) e a Superintendência de Ciências e Tecnologia do Estado de Mato Grosso do Sul (Sucitec/MS) pelo convite de participação neste fascículo especial da Iheringia, Série Zoologia e o suporte financeiro para sua publicação. À Fundação de Apoio e Desenvolvimento do Ensino, Ciência e Tecnologia do Mato Grosso do Sul pelo auxílio financeiro, e à Universidade Federal do Mato Grosso do Sul pelo apoio logístico. A Jaime Luiz Lopes Pereira (NUPELIA/UEM), pela confecção do mapa.

\section{REFERÊNCIAS BIBLIOGRÁFICAS}

Bode, S. N. S.; Adolfson, S.; Bautz, E. R. D.; Martins, M. J. F.; Schmit, O.; Vandekerkhove, J.; Mezquita, F.; Namiotko, T.; Rossetti, G.; Schön, I.; Butlin, R. K. \& Martens, K. 2010. Exceptional cryptic diversity and multiple origins of parthenogenesis in a freshwater ostracod. Molecular Phylogenetics and Evolution 54:542-552.

CHAVES, M.R. 2012. Diversidade e abundância de ostrácodes (Crustacea: Ostracoda) associados à Salvinia Séguier. Monografia, Universidade Estadual de Maringá, Maringá.

Ferreira, C. J. A.; Soriano, B. M. A.; Galdino, S. \& Hamilton, S. K. 1994. Anthropogenic factors affecting waters of the Pantanal wetland and associated rivers in the Upper Paraguay River Basin of Brazil. Acta Limnologica Brasiliensia 5:135-148.

Higuti, J. 2006. Fatores reguladores da biodiversidade de Ostracoda (Crustacea) no vale aluvial no alto rio Paraná. Tese de doutorado. Maringá, Universidade Estadual de Maringá.

Higuti, J.; Velho, L. F. M.; Lansac-Tôha, F. A. \& Martens, K. 2007. Pleuston communities are buffered from regional flood pulses: the example of ostracods in the Paraná River floodplain, Brazil. Freshwater Biology 52:1930-1943.

Higuti, J., Meisch, C. \& Martens, K. 2009a. On Paranacypris samambaiensis n. gen. n. sp. (Crustacea, Ostracoda), the first South American psychrodromid, from the alluvial valley of the Upper Paraná River, Brazil. Journal of Natural History 43:769-783.

Higuti, J.; Lansac-Tôha, F. A.; Velho, L. F. M. \& Martens, K. 2009 b. Biodiversity of non-marine ostracods (Crustacea, Ostracoda) in the alluvial valley of the upper Paraná River, Brazil. Brazilian Journal of Biology 69(Suppl.):661-668.

Higuti, J.; Declerck, S. A. J.; Lansac-Tôha, F. A.; Velho, L. F. M. \& Martens, K. 2010. Variation in ostracod (Crustacea, Ostracoda) communities in the alluvial valley of the upper Paraná River (Brazil) in relation to substrate. Hydrobiologia 644:261-278.

Higuti, J. \& Martens, K. 2012. On a new cypridopsine genus (Crustacea, Ostracoda, Cyprididae) from the Upper Paraná River Floodplain (Brazil). Zootaxa 3391:23-38.

Higuti, J.; Schön, I.; Audenaert, L. \& Martens, K. 2013. On the Strandesia obtusatalelliptica - lineage (Crustacea, Ostracoda, Cyprididae) in the alluvial valley of the upper Paraná River (Brazil), with the description of three new species. Crustaceana 86(2):182-211.

Higuti, J. \& Martens, K. 2014. Five new species of Candoninae (Crustacea, Ostracoda) from the alluvial valley of the Upper Paraná River (Brazil, South America). European Journal of Taxonomy 106:1-36.

Higuti, J. \& MARTENS, K. 2016. Invasive South American floating plants are a successful substrate for native Central African pleuston. Biological Invasions 18:1191-1201 
Horne, D. J.; Cohen, A. \& Martens, K. 2002. Taxonomy, morphology and biology of quaternary and living Ostracoda. In: Holmes J. A. \& CHIVAS, A. R. eds. The Ostracoda: Applications in quaternary research geophysical monograph. Washington D.C., American Geophysical Union. (Geophysical Monograph). Vol. 131, p. 5-36.

MARTENS, K. 1998. Diversity and endemicity of recent non-marine ostracods (Crustacea, Ostracoda) from Africa and South America: a faunal comparison. Verhandlungen der Internationalen Vereinigung fur Theoretische und Angewandte Limnologie 26:2093-2097.

Martens, K. \& Behen, F. 1994. A checklist of the non-marine ostracods (Crustacea, Ostracoda) from the inland waters of South America and adjacent islands. Travaux Scientifiques du Musee National d'Histoire Naturelle de Luxembourg 22:1-81.

Martens, K.; Würdig, N. L. \& Behen, F. 1998. Maxillopoda. Non-marine Ostracoda. In: Young, P. S. ed. Catalogue of Crustacea of Brazil. Rio de Janeiro, Museu Nacional, p. 45-65.

Martens, K.; Schön, I.; Meisch, C. \& Horne, D. J. 2008. Global biodiversity of non-marine Ostracoda (Crustacea). Hydrobiologia 595:185-193.

Matsuda, J. T. 2011. Efeito da complexidade de macrófitas aquáticas sobre a diversidade e abundância de ostrácodes (Crustacea) em um ambiente lêntico da planície de inundação do alto rio Paraná. Monografia. Maringá, Universidade Estadual de Maringá.

Miranda, F. S. 2008. Ocorrência de Ostracoda no médio Rio Doce/ MG e revisão do estado da arte no estudo desse grupo no Brasil. Dissertação de Mestrado. Belo Horizonte, Universidade Federal de Minas Gerais.

Mormul, R. P.; Thomaz, S. M.; Higuti, J. \& Martens, K. 2010. Ostracod (Crustacea) colonization of one native and one non-native macrophyte species of Hydrocharitaceae: an experimental evaluation. Hydrobiologia 644:185-193.

Pereira, L. C. 2010. Composição, abundância e diversidade de ostrácodes (Crustacea) associada à Eichhornia crassipes (SOLMS) em um ambiente lêntico da planície de inundação do alto rio Paraná. Monografia. Maringá, Centro Universitário de Maringá, CESUMAR.

Pinto, R. L. 2007. Taxonomia e biologia de Ostracoda terrestres e semiterrestres do Estado de São Paulo. Tese de doutorado. São Paulo, Universidade de São Paulo.
Pinto, R. L.; Rocha, C. E. F. \& Martens, K. 2003. On two new species of the genus Vestalenula Rossetti \& Martens, 1998 (Crustacea, Ostracoda, Darwinulidae) from semi-terrestrial habitats in São Paulo State (Brazil). Zoological Journal of the Linnean Society 139:305-313.

Pinto, R. L.; Rocha, C. E. F. \& Martens, K. 2004. On the genus Penthesilenula Rossetti \& Martens, 1998 (Crustacea, Ostracoda, Darwinulidae) from (semi-) terrestrial habitats in São Paulo State (Brazil), with the description of a new species. Journal of Natural History 38:2567-2589.

Pinto, R. L.; Rocha, C. E. F. \& Martens, K. 2005a. On the evolution of the genus Microdarwinula Danielopol, 1968 (Ostracoda, Darwinulidae) with the description of a new species from semi-terrestrial habitats in São Paulo State (Brazil). Crustaceana 78:975-986.

Pinto, R. L.; Rocha, C. E. F. \& Martens, K. 2005. On new terrestrial ostracods (Crustacea, Ostracoda) from Brazil, primarily from São Paulo State. Zoological Journal of the Linnean Society 145:145-173.

Pinto, R. L.; Rocha, C. E. F. \& Martens, K. 2008. On the first terrestrial ostracod of the Superfamily Cytheroidea (Crustacea, Ostracoda): description of Intrepidocythere ibipora $\mathrm{n}$. gen. n. sp. from forest leaf litter in São Paulo State, Brazil. Zootaxa 1828:29-42.

Por, F. D. 2000. The Pantanal (Mato Grosso, Brazil) and other "hemiendorheic" basins - paradigms for their conservation. Verhandlungen der Internationalen Vereinigung fur Theoretische und Angewandte Limnologie 27:752-755.

Rocha, C. E. F. \& PoR, F. D. 1998. Preliminary comparative data on the fauna of the pleuston in the Southern Pantanal, Brazil, with emphasis on the microcrustaceans. Verhandlungen der Internationalen Vereinigung fur Theoretische und Angewandte Limnologie 26:2137-2140.

Swarts, F. A. 2000. The Pantanal Understanding and preserving the world's largest wetland. St. Paul, Paragon House. 301p.

Würdig, N. L. \& Freitas, S. M. F. 1988. Distribuição espacial e temporal da comunidade de ostrácodes na lagoa emboaba, Rio Grande do Sul, Brasil. Acta Limnologica Brasiliensia 2:677-700.

Würdig, N. L. \& Pinto, I. D. 2001. Pseudocandona pumilis sp. nov. (Ostracoda). Ecological data and distribution in the Tramandaí lagunar system, RS, Southern Brazil. Nauplius 7:39-51. 
Apêndice I. Lista de espécies de Ostracoda do Pantanal Sul Mato-grossense com as respectivas localidades. A numeração corresponde às localidades elencadas na Tab. I. (N.I., não identificado; R\&P, segundo Rocha \& POR (1998).

\section{CYPRIDIDAE}

Diaphanocypris meridana (Furtos, 1936) Würdig \& Pinto, 1990 R\&P; Loc: 3, 7-9, 11, 13-15, 17, 19, 20, 22-25, 28

Heterocypris sp. Loc: 1, 7, 9, 17, 19, 20, 21

Eucypris sp. Loc: 3,8

Hemicypris sp. Loc: 25,26

Stenocypris major (Braid, 1859) Daday, 1898 R\&P; Loc: 11, 17

Strandesia elliptica (Sars, 1901) Müller, 1912 R\&P

Strandesia tolimensis Roessler, 1990 R\&P; Loc: 8, 10, 28

S. trispinosa (Pinto \& Purper, 1965) Broodbakker, 1983 R\&P

S. lansactohai (Higuti \& Martens, 2012 em Higuti et al. 2012) Loc: 7, 8, 11-13, 17, 19, 20, 22-24, 28

Strandesia sp. 1 n. sp. Loc: 20

Bradleytriebella lineata (Victor \& Fernando, 1981) Savatenalinton \& Martens, 2010 Loc: 11

Cypricercus variabilis (Roessler, 1986) R\&P

Chlamydotheca arcuata (Sars, 1901) Müller, 1912 R\&P

C. deformis Farkas, 1958 Loc: 8, 13

C. colombiensis Roessler, 1985 Loc: 3

C. iheringi (Sars, 1901) Klie, 1930 R\&P; Loc: 7, 8, 12, 13, 28

Cypretta vivacis Würdig \& Pinto, 1993 Loc: 14

Cypretta sp. $1 \mathrm{R} \& \mathrm{P}$

Cypretta n. sp. Loc: $11,14,15$

Cypridopsis vidua (O.F. Müller, 1776) Brady, 1867 R\&P; Loc: 8, 9, 13, 14, 22-24

Syn.: Cypridopsis viduella Sars, $1895 \mathrm{R} \& \mathrm{P}$

C. cf. vidua sp. 2 Loc: 14, 22, 23

Cypridopsis sp.1 R\&P

“Cypridopsis" n. gen. 2 n. sp. Loc: 8-15, 17, 19-24, 26, 28

Paranacypris samambaiensis Higuti et al., 2009 Loc: 13

Potamocypris schubarti Klie, 1940 R\&P

Potamocypris sp. 1 n. sp. Loc: 9-17, 19-22

\section{CANDONIDAE}

Candobrasilopsis brasiliensis Sars, 1901 Loc: 16, 19

Candonidae N.I. R\&P; Loc: 6, 8-10, 12, 17, 19, 22

Cypria pellucida Sars, 1901R\&P

Physocypria schubarti Farkas, 1958 Loc: 3, 7-25, 28

Physocypria sp. 2 n. sp. Loc: 10, 15, 17

\section{LIMNOCYTHERIDAE}

Cytheridella ilosvayi Daday, 1905 R\&P; Loc: 6-17, 19-24, 28

Limnocythere sp. 1 Loc: 11

\section{DARWINULIDAE}

Alicenula serricaudata (Klie, 1935) Loc: 9

Darwinula stevensoni (Brady \& Robertson, 1870) R\&P

Penthesilenula brasiliensis (Klie, 1935) (Syn.: Darwinula africana Klie, 1935) R\&P 